\title{
SOME REMARKS ON THE STRUCTURE OF MACKEY FUNCTORS
}

\author{
J. P. C. GREENLEES AND J. P. MAY \\ (Communicated by Frederick R. Cohen)
}

\begin{abstract}
All Mackey functors over a finite group $G$ are built up by short exact sequences from Mackey functors arising from modules over the integral group rings of appropriate subquotients $W H$ of $G$. The equivariant cohomology theories with coefficients in Mackey functors arising from $W H$-modules admit particularly simple descriptions.
\end{abstract}

Let $G$ be a finite group. The notion of a Mackey functor plays a fundamentally important role in induction theory, both in purely algebraic situations and in equivariant stable homotopy theory. A Mackey functor is a fairly complicated algebraic gadget. We give a quick and elementary structural analysis of Mackey functors, which shows that general Mackey functors are built up inductively out of very simple building blocks. Each building block is constructed in a direct and explicit fashion from an ordinary $W H$-module $V$, where $W H=N H / H$ for some subgroup $H$ of $G$.

Our motivation comes from equivariant cohomology theory, and we analyze the cohomology of $G$-spaces $X$ with coefficients in the cited simple building blocks. In fact, these cohomology groups turn out to be nothing but the homology groups of the obvious cochain complex $\operatorname{Hom}_{W H}\left(C_{*}\left(X^{H}\right), V\right)$.

The results here were obtained as a step in our construction of completions of $G$-spectra at ideals of the Burnside ring [2]. In that application, we were able to prove facts about general Mackey functors by induction starting from the corresponding facts about the Mackey functors constructed from $\mathrm{WH}$-modules, which were relatively easy to prove. We would expect analogous applications in other situations. Actually, Hopkins found an elegant substitute for use of the present theory in the context of [2], but the analysis of Mackey functors should be of independent interest.

Much of this analysis is implicit in Lewis's notes [3], which give a more sophisticated study of the structure of Mackey functors. An analysis similar to ours was arrived at independently by Thévenaz and Webb [5]. We are indebted to both Lewis and Webb for useful discussions of this material.

We begin by recalling Dress's definition of a Mackey functor [1]. Let $G \mathscr{S}$ be the category of finite left $G$-sets and $\mathscr{A} b$ be the category of Abelian groups. A Mackey functor $M$ consists of a contravariant and covariant functor $G \mathscr{S} \rightarrow$

Received by the editors April 5, 1990.

1980 Mathematics Subject Classification (1985 Revision). Primary 55N91, $20 \mathrm{~J} 99$.

Key words and phrases. Mackey functor, coefficient system, Bredon cohomology, transfer. 
$\mathscr{A} b$. The two functors have the same object function, denoted $M$, and $M$ converts disjoint unions to direct sums. For a map $\alpha: S \rightarrow T$ of $G$-sets, let $\alpha^{*}: M(T) \rightarrow M(S)$ and $\alpha_{*}: M(S) \rightarrow M(T)$ denote the contravariant and covariant values of $M$ on morphisms; these are called restriction and transfer maps. It is required that $\alpha^{*} \circ \beta_{*}=\delta_{*} \circ \gamma^{*}$ for pullback diagrams of finite $G$-sets

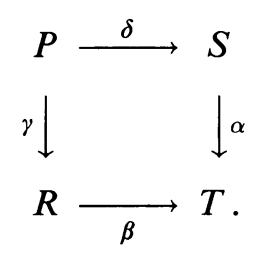

There is a simpler notion of a (Bredon) coefficient system $M$, which is just a contravariant functor $M: G \mathscr{S} \rightarrow \mathscr{A} b$ that converts disjoint unions to direct sums. We have evident notions of morphisms of Mackey functors and of coefficient systems, and we let $\mathscr{M}[G]$ and $\mathscr{B}[G]$ denote the respective categories. We obtain a functor $\mathscr{M}[G] \rightarrow \mathscr{B}[G]$ by forgetting transfer maps. Of course, both Mackey functors and coefficient systems are entirely determined by their behavior on the full subcategory $G \mathscr{O} \subset G \mathscr{S}$ of orbits $G / H$. We let $\mathscr{Z}[G]$ denote the category of left $G$-modules ( $=Z[G]$-modules).

For $H \subset G$, let $N H$ denote the normalizer of $H$ in $G$, and let $W H=$ $N H / H$. (We write $N_{G} H$ and $W_{G} H$ when necessary for clarity.) Let $l: N H \rightarrow$ $G$ and $\varepsilon: N H \rightarrow W H$ be the inclusion and quotient homomorphisms. The basic building blocks for coefficient systems and Mackey functors arise from $W H$-modules, and we start work by describing various functors and adjunctions that help to make the nature of the building blocks clear. We construct the following diagram:

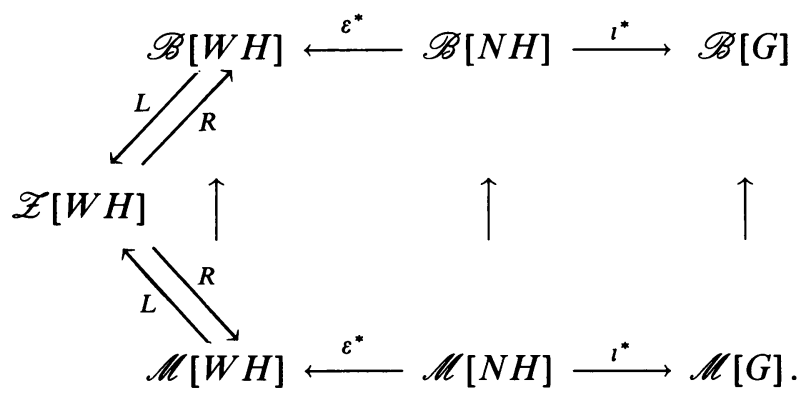

We also construct functors $\varepsilon_{*}$ and $l_{*}$ going in the opposite directions from $\varepsilon^{*}$ and $l^{*}$. By abuse of notation, we continue to write $R$ for its composite with $\varepsilon_{*}$ or with $l^{*} \circ \varepsilon_{*}$ and $L$ for its composite with $\varepsilon^{*}$ or with $\varepsilon^{*} \circ l_{*}$. Mnemonically, $L$ and $R$ stand for left and right adjoints.

Of course, the vertical arrows are the forgetful functors. We focus on the functors involving the bottom row; those involving the top row are similarjust forget about transfer maps. We treat the various functors separately, in their most natural contexts.

Definition 1. Define functors $L: \mathscr{M}[G] \rightarrow \mathscr{Z}[G]$ and $R: \mathscr{Z}[G] \rightarrow \mathscr{M}[G]$ as follows for any finite group $G$. For a Mackey functor $M$, let $L M$ be the $G$-module $M(G / e)$. For a $G$-module $V$, let $(R V)(G / H)=V^{H}$. 
To clarify things later, we write out a full description of $R$ in terms of the definition of a Mackey functor. Define $R V: G \mathscr{S} \rightarrow \mathscr{A} b$ by $(R V)(S)=$ $\left(\bigoplus V_{\sigma}\right)^{G}$, where $V_{\sigma}$ is a copy of $V$ and the sum runs over the set of $G$-maps $\sigma: G \rightarrow S$. An element $g \in G$ specifies a $G$-map $r(g): G \rightarrow G$ via right multiplication by $g$, and $G$ acts on $\bigoplus V_{\sigma}$ by $(g x)_{\sigma}=g \cdot x_{\sigma \circ r\left(g^{-1}\right)}$, where $x_{\sigma}$ denotes the $\sigma$ th coordinate of $x \in \bigoplus V_{\sigma}$. For a map $\alpha: S \rightarrow T$ of $G$-sets, the homomorphisms

$$
\alpha^{*}:(R V)(T) \rightarrow(R V)(S) \text { and } \quad \alpha_{*}:(R V)(S) \rightarrow(R V)(T)
$$

are specified coordinatewise by

$$
\alpha^{*}(y)_{\sigma}=y_{\alpha \circ \sigma} \quad \text { and } \quad \alpha_{*}(x)_{\tau}=\sum_{\alpha \circ \sigma=\tau} x_{\sigma} .
$$

The Mackey functor condition on pullbacks is very easily checked from this description.

Proposition 2. On the level of coefficient systems, the functors $L$ and $R$ are left and right adjoint, so that

$$
\mathscr{Z}[G](L M, V) \cong \mathscr{B}[G](M, R V)
$$

for a G-module $V$ and a coefficient system $M$. This remains true on the level of Mackey functors, so that

$$
\mathscr{Z}[G](L M, V) \cong \mathscr{B}[G](M, R V) \cong \mathscr{M}[G](M, R V)
$$

for a $G$-module $V$ and a Mackey functor $M$.

Proof. Let $\pi: G / e \rightarrow G / H$ be the natural quotient map. The image of $\pi^{*}$ : $M(G / H) \rightarrow M(G / e)$ lies in $M(G / e)^{H}$, so we have a natural map $\eta: M \rightarrow$ $R L M ; \eta$ and the identity $\varepsilon: L R V \rightarrow V$ give the unit and the counit of the adjunction. On general $G$-sets $S, \eta: M \rightarrow R L M$ admits the description $\eta(m)_{\sigma}=\sigma^{*}(m)$ for $m \in M(S)$. The verification that $\eta$ commutes with transfer maps when $M$ is a Mackey functor amounts to an observation about the structure of the pullback of a $G$-map $\tau: G \rightarrow T$ along a $G$-map $\alpha: S \rightarrow T$.

Definition 3. Let $J=G / K$ for a normal subgroup $K$ of a finite group $G$, and let $\varepsilon: G \rightarrow J$ be the quotient homomorphism. Let $\mathscr{M}[G] / K$ be the category of Mackey functors concentrated over $K$; namely, Mackey functors $M$ such that $M(G / H)=0$ unless $H$ contains $K$. Define functors $\varepsilon^{*}: \mathscr{M}[G] \rightarrow \mathscr{M}[J]$ and $\varepsilon_{*}: \mathscr{M}[J] \rightarrow \mathscr{M}[G] / K$ as follows. Let $G \mathscr{S}^{K}$ be the category of $K$-fixed $G$-sets. By regarding a $J$-set $S$ as a $G$-set by pullback along $\varepsilon$, we obtain an isomorphism $\varepsilon^{\#}: J \mathscr{S} \rightarrow G \mathscr{S}^{K}$ with inverse $\varepsilon_{\#}$. For $M \in \mathscr{M}[G]$, composition of $\varepsilon^{\#}$ with $M: G \mathscr{S} \rightarrow \mathscr{A} b$ in both its covariant and contravariant parts gives $\varepsilon^{*} M \in \mathscr{M}[J]$. For $M^{\prime} \in \mathscr{M}[J]$, composition of $\varepsilon_{\#}$ with $M^{\prime}$ in both its covariant and contravariant parts determines $\varepsilon_{*} M^{\prime} \in \mathscr{M}[G] / K$. Visibly $\varepsilon_{*}$ and $\varepsilon^{*}$ establish an isomorphism between $\mathscr{M}[J]$ and $\mathscr{M}[G] / K$.

Proposition 4. On the level of coefficient systems, the functors $\varepsilon^{*}$ and $\varepsilon_{*}$ are left and right adjoint, so that

$$
\mathscr{B}[J]\left(\varepsilon^{*} M, M^{\prime}\right) \cong \mathscr{B}[G]\left(M, \varepsilon_{*} M^{\prime}\right)
$$

for $M \in \mathscr{B}[G]$ and $M^{\prime} \in \mathscr{B}[J]$. Let ${ }_{K} \mathscr{M}[G]$ denote the full subcategory of $\mathscr{M}[G]$ whose objects are those Mackey functors $M$ such that the transfer maps 
$\alpha_{*}: M(G / H) \rightarrow M(G / L)$ associated to those $G$-maps $\alpha: G / H \rightarrow G / L$ with $H \not \supset K$ and $L \supset K$ are zero. On the level of Mackey functors,

$$
\mathscr{M}[J]\left(\varepsilon^{*} M, M^{\prime}\right) \cong{ }_{K} \mathscr{M}[G]\left(M, \varepsilon_{*} M^{\prime}\right)
$$

for $M \in{ }_{K} \mathscr{M}[G]$ and $M^{\prime} \in \mathscr{M}[J]$.

Proof. The first statement is clear. The point of the second statement is that the evident map of coefficient systems $\eta: M \rightarrow \varepsilon_{*} \varepsilon^{*} M$, which is the identity on $M(G / H)$ if $H \supset K$ and is 0 on $M(G / H)$ otherwise, will commute with transfer maps if and only if $M \in K_{K} \mathscr{M}[G]$.

Definition 5. Let $l: H \rightarrow G$ be an inclusion of finite groups. Define functors $l^{*}: \mathscr{M}[H] \rightarrow \mathscr{M}[G]$ and $l_{*}: \mathscr{M}[G] \rightarrow \mathscr{M}[H]$ as follows. By regarding $G$-sets as $H$-sets, we obtain a functor $\imath^{\#}: G \mathscr{S} \rightarrow H \mathscr{S}$. For $M \in \mathscr{M}[H]$, composition of $l^{\#}$ with $M: H \mathscr{S} \rightarrow \mathscr{A} b$ in both its covariant and contravariant parts gives $\imath^{*} M \in \mathscr{M}[G]$. An $H$-set $S$ generates a $G$-set $G \times_{H} S$, and this gives a functor $l_{\#}: H \mathscr{S} \rightarrow G \mathscr{S}$. Composition with $M^{\prime} \in \mathscr{M}[G]$ gives $l_{*} M^{\prime} \in \mathscr{M}[H]$.

Proposition 6. The functors $l_{*}$ and $l^{*}$ are left and right adjoint,

$$
\mathscr{M}[H]\left(l_{*} M^{\prime}, M\right) \cong \mathscr{M}[G]\left(M^{\prime}, l^{*} M\right)
$$

for $M \in \mathscr{M}[H]$ and $M^{\prime} \in \mathscr{M}[G]$, and similarly on the level of coefficient systems.

Proof. The unit $\eta: M^{\prime} \rightarrow l^{*} l_{*} M^{\prime}$ and counit $\varepsilon: l_{*} l^{*} M \rightarrow M$ of the adjunction are induced by the transformations $\xi: l_{\#} l^{\#} \rightarrow$ Id and $\nu:$ Id $\rightarrow l^{\#} l_{\#}$ given by the natural action $G \times{ }_{H} S \rightarrow S$ of $G$ on $G$-sets $S$ and by the natural inclusion of $H$-sets $T$ in $G \times{ }_{H} T$.

Returning to our initial situation with $H, N H, W H$, and $G$, we have the following adjunctions on the coefficient system level:

$$
\begin{aligned}
\mathscr{Z}[W H](L M, V) & \cong \mathscr{B}[W H](M, R V), \\
\mathscr{B}[W H]\left(\varepsilon^{*} M, M^{\prime}\right) & \cong \mathscr{B}[N H]\left(M, \varepsilon_{*} M^{\prime}\right), \\
\mathscr{B}[N H]\left(l_{*} M, M^{\prime}\right) & \cong \mathscr{B}[G]\left(M, l^{*} M^{\prime}\right) .
\end{aligned}
$$

All of the functors in sight are also defined on the Mackey functor level, where the first and third adjunctions remain valid but where the second adjunction must be weakened to

$$
\mathscr{M}[W H]\left(\varepsilon^{*} M, M^{\prime}\right) \cong{ }_{H} \mathscr{M}[N H]\left(M, \varepsilon_{*} M^{\prime}\right) .
$$

If $H_{\mathscr{M}}[G]$ is the full subcategory of $\mathscr{M}[G]$ consisting of those Mackey functors $M$ whose transfer maps $\alpha_{*}: M(G / J) \rightarrow M(G / L)$ associated to $G$-maps $\alpha: G / J \rightarrow G / L$ with $J \not \supset H$ and $L \supset H$ are zero, then $l_{*}$ carries $H_{\mathscr{M}}[G]$ to $H^{\mathscr{M}}[N H]$. We summarize matters in the following proposition. Remember that we have agreed to write

$$
R: \mathscr{Z}[W H] \rightarrow \mathscr{M}[G] \text { and } L: \mathscr{M}[G] \rightarrow \mathscr{Z}[W H]
$$

for the composite functors $l^{*} \varepsilon_{*} R$ and $L \varepsilon^{*} l_{*}$. For a $W H$-module $V,(R V)(G / H)$ $=V$ and $(R V)(G / J)=0$ unless $H$ is conjugate to a subgroup of $J$. For a $G$-Mackey functor $M, L M=M(G / H)$. 
Proposition 7. On the level of coefficient systems, the functors $L$ and $R$ are left and right adjoint, so that

$$
\mathscr{Z}[W H](L M, V) \cong \mathscr{B}[G](M, R V)
$$

for a $W H$-module $V$ and a $G$-coefficient system $M$. The counit $\varepsilon: L R V=$ $(R V)(G / H) \rightarrow V$ of the adjunction is an isomorphism. On the level of Mackey functors, there is a restricted adjunction

$$
\mathscr{Z}[W H](L M, V) \cong{ }_{H} \mathscr{M}[G](M, R V) .
$$

In particular, if $M \in{ }_{H} \mathscr{M}[G]$, then the identity map of $M(G / H)$ extends uniquely to a map of Mackey functors $\eta: M \rightarrow R(M(G / H))$.

Tracing through the definitions, we see that, for a $G$-set $S,(R V)(S)=$ $\left(\bigoplus V_{\sigma}\right)^{W H}$, where the sum runs over the set of $W H$-maps $\sigma: W H \rightarrow S^{H}$ (or, equivalently, the set of $G$-maps $G / H \rightarrow S$ ); of course, $(R V)(S)=0$ if $S^{H}$ is empty. The restriction and transfer maps associated to a $G$-map $\alpha: S \rightarrow T$ are obtained from the induced $W H$-map $\alpha^{H}: S^{H} \rightarrow T^{H}$ in the fashion described earlier. The inverse $V \rightarrow(R V)(G / H)$ of the counit $\varepsilon$ sends an element $v \in V$ to the element with $\sigma$ th coordinate $w v$, where $\sigma: W H \rightarrow(G / H)^{H}=W H$ is right multiplication by $w \in W H$.

From our point of view, the crucial simplifying feature of the Mackey functors $R V$ is that the action of the Burnside ring $A(G)$ takes a particularly simple form on them. Additively, $A(G)$ is a free Abelian group with basis elements $[G / H]$, where $H$ runs over a set of representatives for the conjugacy classes of subgroups of $G$. For a Mackey functor $M$, the action of $[G / H]$ on $M(S)$ is the composite $\pi_{*} \circ \pi^{*}$, where $\pi$ is the projection $S \times G / H \rightarrow S$. Recall that there is a homomorphism of rings $\varphi_{H}: A(G) \rightarrow Z$, which sends a finite $G$-set $S$ to the cardinality of $S^{H}$.

Proposition 8. If a Mackey functor $N$ satisfies $N(G / J)=0$ for all proper subgroups $J$ of $H$, then $A(G)$ acts through $\varphi_{H}$ on $N(G / H)$. If $N=R V$ for a $W H$-module $V$, then $A(G)$ acts through $\varphi_{H}$ on $N(S)$ for all finite $G$-sets $S$. Proof. $A(G)$ acts on $N(G / H)$ through the restriction $r: A(G) \rightarrow A(H)$, and $\varphi_{H} \circ r=\varphi_{H}$. The action of $[H / J]$ on $N(G / H)$ is the composite of the restriction $N(G / H) \rightarrow N(G / J)$ and the transfer $N(G / J) \rightarrow N(G / H)$ and is thus zero unless $J=H$, when it is the identity. It follows that $\alpha x=\varphi_{H}(\alpha) x$ for $\alpha \in A(G)$ and $x \in N(G / H)$. If $N=R V$ for a $W H$-module $V$, then direct computation shows that the composite

$$
N(S) \stackrel{\pi^{*}}{\longrightarrow} N(G / K \times S) \stackrel{\pi_{*}}{\longrightarrow} N(S)
$$

is multiplication by the cardinality of $(G / K)^{H}$ for any $K \subset G$. To see this, let $y \in\left(\bigoplus V_{\sigma}\right)^{W H}$, where the sum runs over the $W H$-maps $\sigma: W H \rightarrow S^{H}$. Then

$$
\left(\pi_{*} \pi^{*}(y)\right)_{\sigma}=\Sigma \pi^{*}(y)_{\nu}=\Sigma y_{\pi \circ \nu}=\Sigma y_{\sigma},
$$

where the sums run over the $W H$-maps $\nu: W H \rightarrow(G / K)^{H} \times S^{H}$ such that $\pi \circ \nu=\sigma$; clearly there are $\left|(G / K)^{H}\right|$ such maps $\nu$.

The coefficient system level adjunction of Proposition 7 leads to a simple algebraic description of Bredon cohomology with coefficients in $R V$. 
Proposition 9. Let $X$ be a $G-C W$ complex. For a $W H$-module $V$, the Bredon cohomology $H_{G}^{*}(X ; R V)$ is naturally isomorphic to the cohomology of the cochain complex $\operatorname{Hom}_{W H}\left(C_{*}\left(X^{H}\right), V\right)$. It is therefore also isomorphic to the Bredon cohomology $H_{W H}^{*}\left(X^{H} ; R V\right)$.

Proof. The cellular chain complex $\underline{C}_{*}(X)$ in the Abelian category of coefficient systems is specified on orbits by $\underline{C}_{*}(X)(G / H)=C_{*}\left(X^{H}\right)$. In Hom notation, the adjunction of Proposition 7 specializes to give an isomorphism of cochain complexes

$$
\operatorname{Hom}_{W H}\left(C_{*}\left(X^{H}\right), V\right) \cong \operatorname{Hom}_{\mathscr{B}[G]}\left(\underline{C}_{*}(X), R V\right) \text {. }
$$

By definition, the cohomology of the right-hand side, and thus of the left-hand side, is the Bredon cohomology $H_{G}^{*}(X ; R V)$. The last statement follows by applying the first statement to the $W H-C W$ complex $X^{H}$ and the functor $R: \mathscr{Z}[W H] \rightarrow \mathscr{M}[W H]$.

For the aficionados, we record the following much less obvious spectrum level analog of the last statement. (See [4, II §9] for the relevant definitions.)

Proposition 10. For a G-spectrum $X$ and $W H$-module $V$,

$$
H_{G}^{*}(X, R V) \cong H_{W H}^{*}\left(\Phi^{H} X, R V\right) \text {. }
$$

In particular, if $\pi_{*}\left(X^{K}\right)=0$ for those subgroups $K$ of $N H$ that do not contain $H$, then

$$
H_{G}^{*}(X, R V) \cong H_{W H}^{*}\left(X^{H}, R V\right) .
$$

Proof. Consider the Eilenberg-MacLane $W H$-spectrum $K(R V, 0)$. Its associated $N H$-spectrum concentrated over $H$ is defined by applying the functor $\widetilde{E} \mathscr{F}[H] \wedge \varepsilon^{\#}(?)$ of $[4$, II $\S 9]$ and is an Eilenberg-MacLane spectrum $K\left(\varepsilon_{*} R V, 0\right)$. The $G$-spectrum generated by this $N H$-spectrum is defined by applying the functor $F_{N H}[G, ?)$ of [4, II.4.1] and is an Eilenberg-MacLane spectrum $K\left(l^{*} \varepsilon_{*} R V, 0\right)$. The conclusion follows from the general change of groups isomorphisms of $[4$, II.4.3, II.9.2, II.9.5].

We should also record the rather standard fact that Proposition 9 implies the presence of a well-behaved transfer homomorphism.

Proposition 11. Let $X$ be a $G-C W$ complex. For $J \subset H \subset G$ and $a W_{G} J$ module $V$, there is a transfer homomorphism

$$
\tau: H_{H}^{*}(X ; R V) \rightarrow H_{G}^{*}(X ; R V)
$$

whose composite $\tau \circ \pi$ with the forgetful homomorphism

$$
\pi: H_{G}^{*}(X ; R V) \rightarrow H_{H}^{*}(X ; R V)
$$

is multiplication by the index $\left[N_{G} J: N_{H} J\right]$ of normalizers.

Proof. For a $W_{H} J$-homomorphism $f: C_{*}\left(X^{J}\right) \rightarrow V$, define the $W_{G} J$-homomorphism $\tau f: C_{*}\left(X^{J}\right) \rightarrow V$ to be the sum over coset representatives $g$ of $N_{H} J$ in $H_{G} J$ of the homomorphisms $g f$, where $g$ acts by conjugation on Hom groups. Then $\tau$ is a well-defined map of cochain complexes, and the composite $\tau \circ \pi$ is multiplication by $\left[N_{G} J: N_{H} J\right]$ on the cochain level.

Finally, the following result and its proof describe how to construct arbitrary Mackey functors out of the Mackey functors $R V$. 
Theorem 12. Every Mackey functor is built from Mackey functors of the form $R V$. Precisely, if $\mathscr{A}$ is the smallest collection of Mackey functors that satisfies the following closure properties, then every Mackey functor is in $\mathscr{A}$.

(1) $R V$ is in $\mathscr{A}$ for all $W H$-modules $V$ and all subgroups $H$ of $G$.

(2) If $0 \rightarrow N^{\prime} \rightarrow N \rightarrow N^{\prime \prime} \rightarrow 0$ is an exact sequence of Mackey functors such that two of $N, N^{\prime}$, and $N^{\prime \prime}$ are in $\mathscr{A}$, then so is the third.

Proof. Partition the set of subgroups of $G$ as follows. Let $S_{0}=\{e\}$. Inductively, let $S_{j}$ consist of those subgroups that are not in $S_{j-1}$ but each of whose proper subgroups is in $S_{i}$ for some $i<j$. There is an $m$ such that $S_{m}=\{G\}$. Eachi $S_{j}$ is closed under conjugation, say with $n_{j}$ conjugacy classes. Choose a subgroup $H_{j, k}$ in each conjugacy class, $1 \leq k \leq n_{j}$. Say that a Mackey functor $M$ is of type $(j, k)$ if $M\left(G / H_{j, k}\right) \neq 0$ but $M\left(G / H_{j^{\prime}, k^{\prime}}\right)=0$ for $j^{\prime}<j$ and for $j^{\prime}=j$ and $k^{\prime}<k$. If $M(G / H)=0$ for all proper subgroups $H$, then $M=R M(G / G)$. Inductively, let $M$ be of type $(j, k)$ and assume that all Mackey functors of type $\left(j^{\prime}, k^{\prime}\right)$ with $j^{\prime}>j$ or $j^{\prime}=j$ and $k^{\prime}>k$ are in $\mathscr{A}$. We have a map $\eta: M \rightarrow R M\left(G / H_{j, k}\right)$ of Mackey functors that is the identity on $M\left(G / H_{j, k}\right)$. The induction hypothesis clearly applies to show that $\operatorname{Coker}(\eta)$ and $\operatorname{Ker}(\eta)$ are both in $\mathscr{A}$. Two applications of the second closure property imply that $\operatorname{Im}(\eta)$ and $M$ are also in $\mathscr{A}$.

\section{BIBLIOGRAPHY}

1. A. Dress, Contributions to the theory of induced representations, Lecture Notes in Math., vol. 342, Springer-Verlag, New York, 1973, pp. 183-240.

2. J. P. C. Greenlees, M. J. Hopkins, and J. P. May, Completions of G-spectra at ideals of the Burnside ring. I, preprint.

3. L. G. Lewis, Jr., The theory of Green functors, mimeographed notes.

4. L. G. Lewis, Jr., J. P. May, and M. Steinberger, Equivariant stable homotopy theory, Lecture Notes in Math., vol. 1213, Springer-Verlag, New York, 1986.

5. J. Thévenaz and P. J. Webb, Simple Mackey functors, Proc. of the 1989 Bressanone Conf. on Group Theory (to appear).

Department of Mathematics, The University of Chicago, Chicago Illinois 60637

Current address: (J. P. C. Greenlees) Department of Mathematics, Sheffield University, Sheffield, England 